\title{
Secondary Intraocular Lens Implantation: Eight Year Experience
}

\author{
J. MICHAEL HAYWARD, BRUCE A. NOBLE, NICHOLAS GEORGE \\ Leeds
}

\begin{abstract}
Summary
Objective-To investigate the outcome of secondary intra-ocular lens implantation. Neither a requirement for vitreous surgery nor previous complicated eye surgery/ trauma was considered necessarily to be a contra-indication.

Design-Retrospective Study. Recruitment was achieved by examination of operating theatre records.

Setting-Teaching Hospital.

Patients-52 out of 54 consecutive cases in whom a secondary lens had been implanted from 1982-1989 under the care of one consultant (BAN).

Interventions-The medical records of all patients were reviewed and data collected for 52 of the total of 54 patients.

Main outcome measures-Visual acuity and major complications.

Results $-77 \%$ of eyes retained the same Snellen visual acuity or better than that pre-operatively and $88 \%$ were within one line or better. The Mann Whitney U-test showed that neither vitreous manipulation at the time of secondary lens implantation nor a previous history of trauma had a significant effect on the change between preand post-operative Snellen visual acuity. Only one patient developed a retinal detachment and eight had cystoid macula oedema although in only one patient did this account for a final decrease in post-operative visual acuity of more than one line. The most common complication was an intense post-operative uveitis $(35 \%)$ leading in some cases to indefinite low dose topical steroid treatment $(21 \%)$.

Conclusions-This technique gives good visual results, even in the disorganised eye, providing the vitreous is adequately managed. However there is a significant complication rate which should be discussed with the patient before undertaking surgery.
\end{abstract}

\section{Introduction}

Secondary intraocular lens (IOL) implantation provides an important alternative to aphakic spectacles or contact lenses for aphakic correction and is now a routine ophthalmic procedure. However, for some surgeons, ${ }^{1}$ the presence of vitreous in the anterior chamber, or other anterior segment disruption, is considered to be a contra-indication. Such patients are thus denied useful visual rehabilitation; is this caution justified?

\section{Subjects and Methods}

We reviewed the notes of all patients undergoing secondary lens implantation by one firm at Leeds General Infirmary from 1 January 1982 to 31 July 1989 . Two out of the 54 consecutive cases identified were excluded from the series; one patient was severely mentally retarded, another defaulted from follow-up after two months. The cases could be divided into three categories as shown in Table I. The incidence of secondary lens implantation over 
Table I Groups

\begin{tabular}{llll}
\hline Group & Previous Surgery & $\begin{array}{l}\text { Vitreous Manipulation } \\
\text { at } 2^{\circ} \text { Lens Implantation }\end{array}$ & No. \\
\hline 1 & Senile cataract & No & 22 \\
2 & Senile or Traumatic cataract & Yes & 18 \\
3 & Traumatic cataract & No & 12 \\
\hline
\end{tabular}

this period is shown in Figure 1 . The mean (and range) ages for the groups were 69 (40$84), 66(27-83)$ and $29(5-52)$ years respectively. and mean follow-up times were $26(6-$ 59), 31 (6-68), 28 (8-72) months. These are almost twice as long as some other series where this has been documented. ${ }^{1,6,7,9}$ Four cases from Group 1, six from Group 2 and one from Group 3 had either been discharged from the clinic, moved away or otherwise lost to follow-up at mean post-implantation times of 18,25 and 26 months respectively. Previous cataract surgery is shown in Table II and the mean time that had elapsed since the previous surgical procedure to the eye was two years (0.25-19), four years (0.75-34) and one year $(0.25-41)$ respectively for the three groups. The indications (more than one possible for each case) for secondary lens implantation were aphakic spectacle intolerance, 11 cases $(21 \%)$, contact lens intolerance (either patient and/or eye intolerance) 20 cases $(39 \%)$, unilateral aphakia (contact lens not tried) 17 cases (33\%), ocular motility disorder eight cases (seven from Group 3) (15\%), HGV licence retention one case $(2 \%)$ and hypermetropic surprise one case $(2 \%)$.

The basic surgical technique usually consisted of a corneal section, anterior vitrectomy (if required) with the use of an ocutome cutting suction apparatus (11 cases) or, in some of the earlier cases (7), division of fine vitreous strands with scissors and sponges, implantation of the IOL and closure with a non-absorable monofilament nylon suture. Healonid and subconjuctival antibiotic and

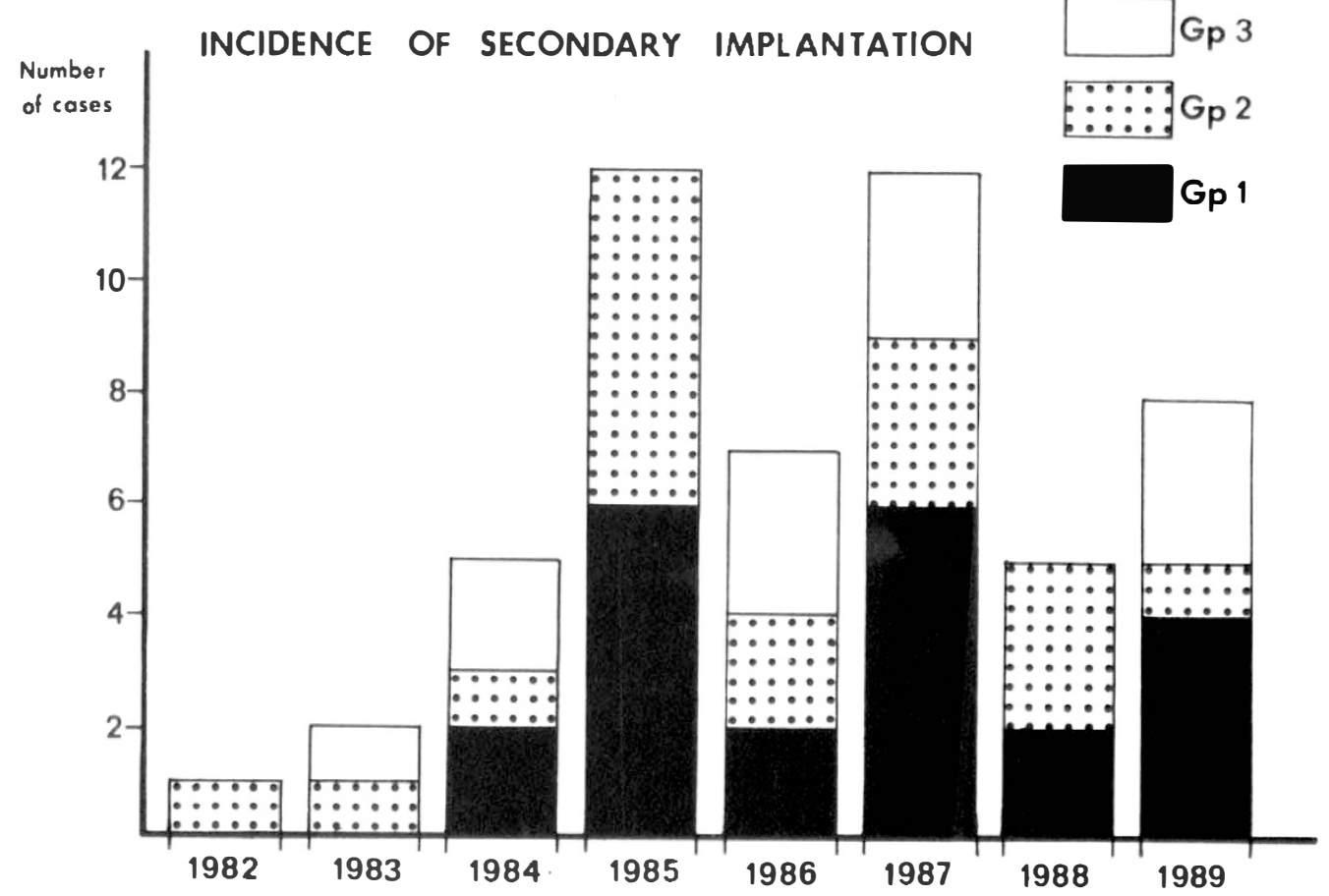

Fig. 1. Incidence of secondary lens implantation in this series. 
Table II Previous cataract surgery

\begin{tabular}{lccc}
\hline & Group 1 & Group 2 & Group 3 \\
\hline ICCE & 9 & 13 & 1 \\
ECCE & 7 & 2 & 4 \\
ICCE/ECCE + Anterior Vitrectomy & 6 & 1 & 2 \\
Lens Aspiration & & 1 & 5 \\
Needling & & 1 & 5 \\
Lensectomy & & \\
\hline
\end{tabular}

steroid were used routinely. Specular microscopy has only just become available to the department and was not performed on patients in this study. Biometry became available in 1986; prior to this standard power lenses were used. The lens types used were Cilco Mutiflex AC lens 48 cases (92\%), Pharmacia J Loop PC lens one case, Cilco SK21 PC lens two cases and Pharmacia Noble PC lens one case.

\section{Results}

\section{Visual Acuity}

The visual results are expressed in percentage terms in Table III. A comparison of corrected pre-operative VA and the last recorded corrected post-operative VA (NB not necessarily best post-operative VA) is shown in Figures $2-4$. Post-operative visual acuity was within one Snellen line or better in $86 \%$ of Group 1, $94 \%$ of Group 2 and $83 \%$ of Group 3 .

For the purposes of statistical comparison the two trauma cases (whose vision had improved by two and four lines Snellen) were removed from group 2 so that this group could be directly compared with Group 1 (i.e. senile cataract cases only). The upper and lower quartiles and median values of the change in visual acuity (in terms of lines Snellen) are shown in Figure 5. A one tailed Mann Whitney U-test using the normal approximations and allowing for tied rank, gave $\mathrm{z}$ values of -0.66 (Group 1 v Group 2) and -0.04 (Group 1 v Group 3) where a value above +1.64 would be significant at the $5 \%$ level

Table III Post-operative Snellen Visual Acuity

\begin{tabular}{lrrr}
\hline VA & Group 1 & Group 2 & Group 3 \\
\hline Worse by 2 lines or more & $14 \%$ & $6 \%$ & $17 \%$ \\
Worse by 1 line & $9 \%$ & $17 \%$ & $8 \%$ \\
No change & $41 \%$ & $22 \%$ & $25 \%$ \\
Better & $36 \%$ & $55 \%$ & $50 \%$ \\
\hline
\end{tabular}

shows that in both comparisons Group 1 was slightly worse than the other groups. This difference would not have been found significant using a two tailed test.

It should be noted that four patients experienced a reduction in best post-operative visual acuity 1-3 years after surgery (Table IV). In all cases this reduction was two or more lines Snellen and accounted for $4 / 6$ cases of post-operative visual loss of this magnitude. The other cases were caused by increased myopic degeneration problem with refraction and another case of post-operative astigmatism requiring refractive surgery. The median time to achieve best visual acuity was for Group I two months (1-36), Group 2 two months (1-12) and Group 3 three months (118). Ten cases took longer than two months to achieve best visual acuity and the causes of this were cystoid macular oedema (8), treatment of amblyopia (1) and posterior capsule opacity (1).

\section{Complications}

Cystoid Macular Oedema: (CMO)

Eight cases (15\%) had CMO and four of these were known to have had this complication before surgery. The results for these patients

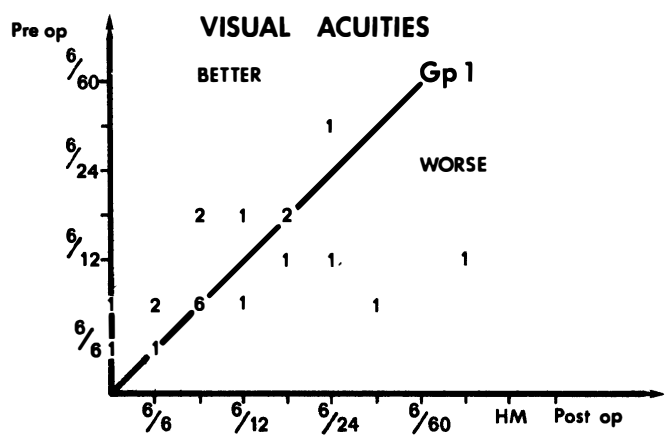

Fig. 2. Comparison of best corrected pre-operative visual acuity and last recorded visual acuity for Group 1. 


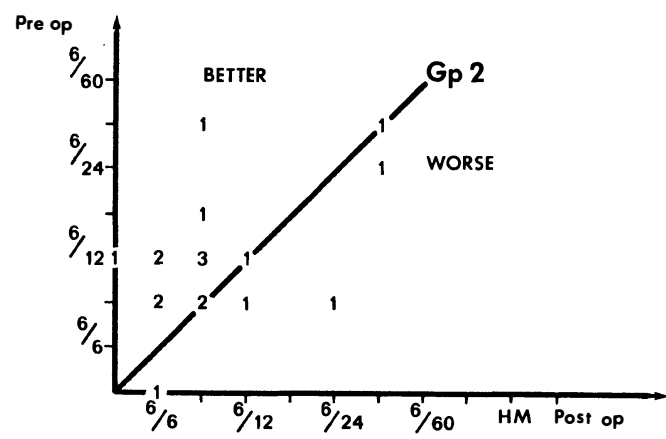

Fig. 3 Comparison of best corrected pre-operative visual acuity and last recorded visual acuity for Group 2.

are shown in Table V. In only one case did it cause a reduction in vision of two or more lines Snellen but the time taken to achieve best post-operative visual acuity was in most cases considerably longer compared to cases without this complication. Groups 2 and 3 did not seem to have a propensity for this complication and of the four cases that had evidence of this complication following previous eye surgery two retained pre-operative visual acuity.

\section{Retinal Detachment}

One case occurred in Group 2 three years after surgery. This eye had vitreous incarcerated in the anterior chamber after secondary lens implantation. The detachment was successfully repaired (but the vision is limited

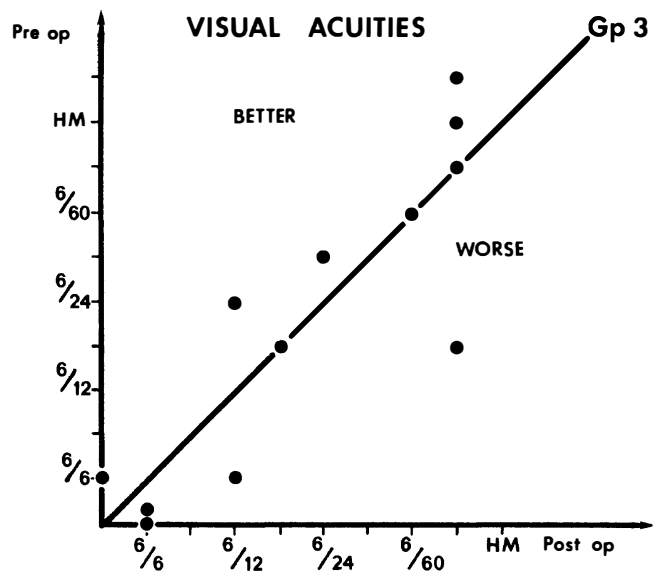

Fig. 4. Comparison of best corrected pre-operative visual acuity and last recorded visual acuity for Group 3. due to pre-detachment, and indeed, pre-lens implantation, $\mathrm{CMO}$ ).

\section{Corneal Decompensation}

One case occurred in Group 1 three years following surgery in an eye where the initial cataract surgery had, to say the least, not been ideal. She is awaiting a corneal graft.

\section{Uveitis}

Intense post-operative inflammation (anterior chamber cells ++ and/or lens precipitates) was the most common complication $(35 \%)$, particularly in the trauma group $(50 \%)$. This was the only complication where the numbers are large enough for statistical comparison. The $\chi^{2}$ test showed that the difference between the groups was not signifi-

\begin{tabular}{c|ccc} 
& \multicolumn{3}{|c}{ Group } \\
& 1 & 2 & 3 \\
\hline Upper quartile & +1 & +1 & +1 \\
Median & 0 & +.5 & 0 \\
Lower quartile & -25 & $-\cdot 75$ & -1
\end{tabular}

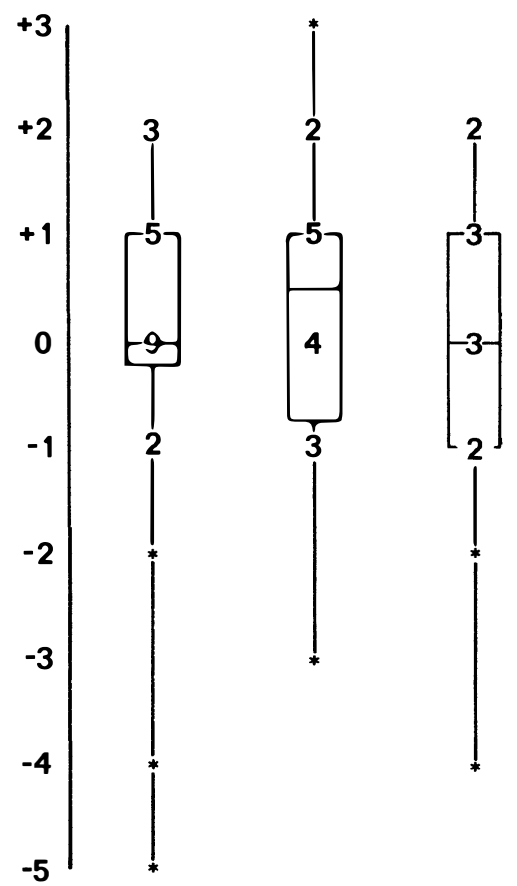

Fig. 5 Statistical comparison of the difference (in lines Snellen) between pre-operative visual acuity and last recorded visual acuity for the three groups. 
Table IV Patients experiencing reduction in best post-operative visual acuity (years after surgery and cause)

\begin{tabular}{lll}
\hline Group 12 years & $6 / 9 \rightarrow 6 / 36$ & Patient co-operation with refraction \\
Group 13 years & $6 / 9 \rightarrow \mathrm{CF}$ & Corneal Decompensation \\
Group 2 2 years & $6 / 9 \rightarrow 6 / 18$ & CMO \\
Group 3 1 year & $6 / 18 \rightarrow \mathrm{CF}$ & Irregular astigmatism \\
\hline
\end{tabular}

cant (Table VI). Twenty-one per cent of patients still require once daily application of a topical steroid for control: 5/22 Group 1 (mean post-operative time 20 months), 5/18 Group 2 (45 months) and 1/12 Group 3 (21 months). One of the cases still requiring treatment had had a posterior chamber lens inserted; this was the only complication seen with the four cases that had insertion of a posterior chamber lens all of which retained or improved on pre-operative visual acuity.

\section{Glaucoma}

Three cases developed ocular hypertension post-operatively but in one case treatment was no longer required after six months.

The two other cases are both being treated with topical medication. Four cases had glaucoma pre-operatively, the effect of surgery on their control is shown in Table VII. The fourth case shown is that of a 25 year old man who suffered a most vicious attack on his head and eye with a golf club producing lid lacerations, a hyphaema, sphincter pupillae damage and a subluxed lens. Following a lensectomy he developed a total hyphaema and then an uncontrolled rise in intra-ocular pressure which eventually resolved following the insertion of a Molteno Tube (Fig. 6). Because of macular trauma his pre- and post-operative (Fig. 7) visual acuity is limited to around 6/60

Table V Cystoid Macular Oedema GRP Preoperative VA Months to Best VA Last Recorded VA

\begin{tabular}{cccc}
\hline GRP & $\begin{array}{c}\text { Pre-op } \\
\text { VA }\end{array}$ & $\begin{array}{c}\text { Months to } \\
\text { best VA }\end{array}$ & $\begin{array}{c}\text { Last } \\
\text { recorded } V A\end{array}$ \\
\hline Cases with pre-op & CMO & & \\
1 & $6 / 18$ & 6 & $6 / 9$ \\
1 & $6 / 18$ & 3 & $6 / 18$ \\
2 & $6 / 24$ & 6 & $6 / 36$ \\
3 & $4 / 60$ & 3 & $\mathrm{CF}$ \\
Cases without pre-op CMO & & \\
1 & $6 / 18$ & 6 & $6 / 12$ \\
1 & $6 / 18$ & 36 & $6 / 18$ \\
2 & $6 / 9$ & 6 & $6 / 18$ \\
2 & $6 / 9$ & 6 & $6 / 9$ \\
\hline
\end{tabular}

but he is delighted with the increased focus of his peripheral vision and, $2 \frac{1}{2}$ years after the initial injury, and one year after secondary lens implantation, shows no signs of secondary divergence. The function of the Molteno Tube was not affected by lens implantation.

\section{Mobile AC lens}

Four cases had lenses that were not totally stable but none of these had gross movement of the lens. None of the patients experienced lens induced pain and no lenses have had to be removed.

\section{Vitreous}

Of the 11 cases in Group 2 in whom a formal anterior vitrectomy had been performed only one had vitreous incarceration in the anterior chamber/wound post-operatively; this case went on to develop a retinal detachment (vide supra). Of the seven who had vitreous strands cut with scissors \pm sponges, four had strands still remaining in the anterior chamber/ wound. One of these had mild CMO but with retention of $6 / 9$ vision. No case from Groups 1

Table VI Post-operative Uveitis-Statistical comparison between Groups

\begin{tabular}{lccc}
\hline & & Group 1 v & Group 2 \\
\hline \multirow{3}{*}{ Post-operative uveitis } & Ges & Group 1 & Group 2 \\
& No & 17 & 6 \\
\cline { 2 - 3 } & & 22 & 10 \\
\hline
\end{tabular}

Group 2 is slightly worse than Group 1 with an $\chi^{2}$ value of 0.40 (on one degree of freedom) which is not significant.

\begin{tabular}{lccc}
\hline & & \multicolumn{2}{c}{ Group 1 v } \\
& & \multicolumn{2}{c}{ Group 3 } \\
\hline \multirow{3}{*}{ Post-operative uveitis } & Yes & 5 & 6 \\
& No & 17 & 6 \\
\cline { 2 - 3 } & & 22 & 12 \\
\hline
\end{tabular}

Although the difference between the groups (23\% and $50 \%$ ) is greater, within group 3 worse than group 1 , the difference is again not significant with an $\chi^{2}$ value of 1.54 . 
Table VII Glaucoma

\begin{tabular}{lll}
\hline Group & Pre-operative control & 4 Cases with \\
\hline 1 & Tlaucoma pre-op & Post-operative control \\
1 & Topical & Molteno tube + Topical \\
1 & Topical & Topical \\
3 & Molteno Tube & Trabeculectomy \\
\hline
\end{tabular}

Table VIII Final Visual Acuity Comparison with Other Series

\begin{tabular}{lccccc}
\hline Snellen $V A$ & Leeds & Wong & Lindstrom & Shammas & Leatherbarrow \\
\hline Worse by 2 lines & 12 & 4 & 9 & 3 & 15 \\
Worse & 23 & & & 42 & 40 \\
Same & 31 & 72 & 65 & 55 & 82 \\
Same \pm line & 67 & 24 & 26 & 3 \\
Better & 46 & 21 & & & 3 \\
Better by 2 lines & & & & \\
\hline
\end{tabular}

or 3 had vitreous in the anterior chamber post-operatively.

\section{Ocular Motility}

Eight (seven from Group 3) of our patients had a pre-operative disorder of ocular motility. Only one had diplopia post-operatively. This was following squint surgery (with adjustable sutures). However he was very happy with the overall result of surgery (Figures 8-10). Four had BSV post two degree IOL (one required squint surgery). One had better control of an intermittent exotropia. Of the two children one still has profound amblyopia and a divergent eye but the other has had successful occlusion in reversing amblyopia and following squint surgery has a small esotropia with suppression.

\section{Refraction}

The change in cylindrical correction is shown

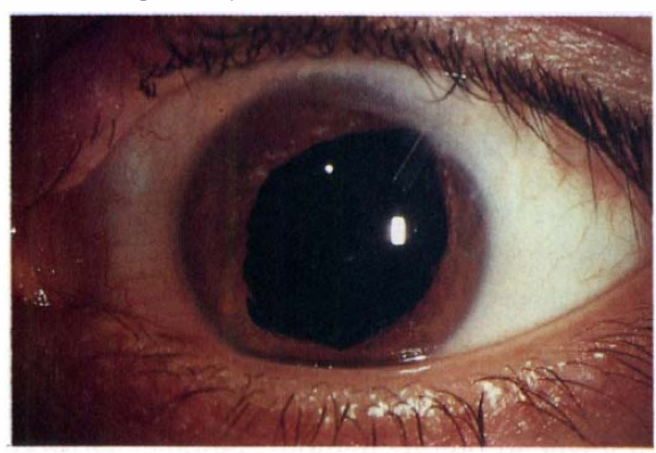

Fig. 6 Case report: Sequelae of blunt trauma: Molteno tube inserted. in Figure 11. Five cases out of the nine that either had an increase $\gg 1$ dioptre or residual cylinder $>3$ dioptres had had an inappropriate placement of incision for the prevailing pre-operative axis of cylinder. Two cases from Group 3 are awaiting further refractive surgery. Seven patients had a post-operative spherical correction of -4.00 dioptres or greater $(-4.00--9.75)$ although only three of these were rendered significantly anisometropic. Six procedures had been performed before biometry and a wide range of lens powers were routinely available in the department. The highest myopic correction was a planned one at -9.75 in a patient who had had a 'myopic surprise' following ECCE + IOL on the other eye. Although myopic he has only half a dioptre anisometropia.

\section{Discussion}

Because of other demands on our Depart-

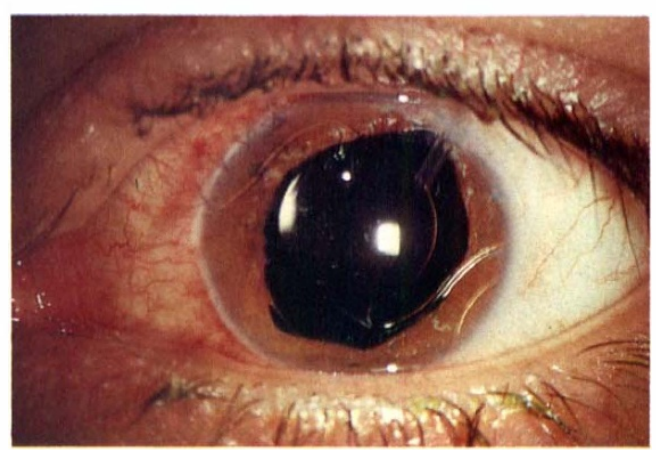

Fig. 7 Same eye as Figure 6 post lens implantation. 


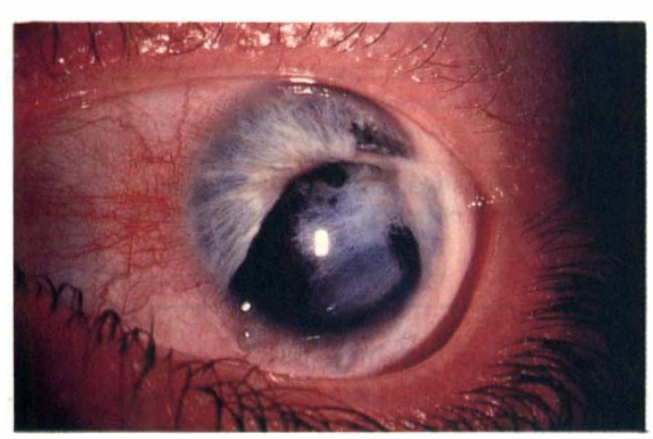

Fig. 8 Case report: Eye that sustained a penetrating injury many years previously.

ment's time and resources this is a small series. However the results stand up to comparison with other reported series as shown in Table VIII. Our results are shown for the three groups together. Although such comparisons are limited due to the differing nature of the cases and single/multiple surgeons involved, our results give us confidence to continue to implant secondary lenses, not only in the uncomplicated eye, but also in the eye with a disordered anterior segment. There is however a significant complication rate about which patients must be forewarned and indeed to which surgeons must be prepared to respond.

Cystoid Macula Oedema (CMO) occurred in eight cases $(15 \%)$. Four of these had had evidence of this complication following previous eye surgery; two retained pre-operative visual activity and two lost one line Snellen. Although active CMO should be treated before further surgery, we do not believe a history of past CMO should necessarily be a

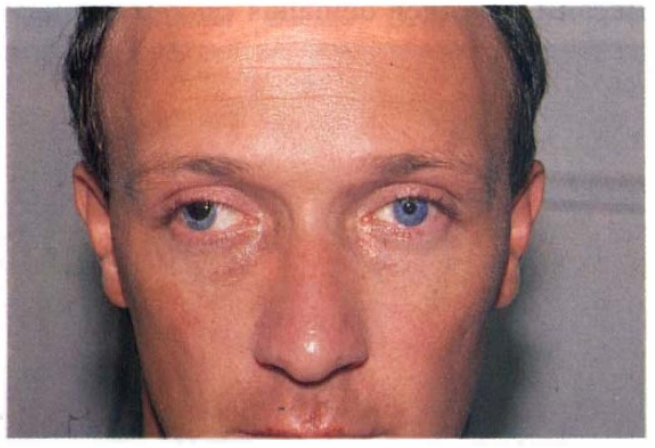

Fig. 9 Same eye (right) as Figure 8 post lens implantation: note divergent squint. contra-indication to secondary lens implantation. Indeed it could be argued that in the presence of a vitreous wick, anterior vitrectomy is an appropriate treatment for $\mathrm{CMO}^{2}$ The only eye to lose two or more lines Snellen due to this complication had no prior history of CMO. Groups 2 and 3 did not seem to have a propensity for this condition. Even patients with an intact hyaloid face are not immune from this complication although it has been reported to be a favourable condition. ${ }^{3}$ Others ${ }^{4}$ have shown that having an interval of at least one year between cataract surgery and secondary IOL insertion may reduce the incidence of CMO and $80 \%$ of all our patients fell into this interval category.

Wong ${ }^{5}$ reported a higher incidence of retinal detachment in cases requiring anterior vitrectomy. Our overall incidence of retinal complications was $17 \%$ and that of Group 2 alone $22 \%$, both lower than the $28 \%$ reported by Wong.

Our one detachment did occur in this group but in a patient in whom a vitreous wick was present both after the initial cataract surgery and also, to a lesser extent, after secondary IOL implantation. This emphasises the need for dealing with the vitreous adequately when performing this surgery. Our results show the importance of performing a formal closed mircosurgical anterior vitrectomy when required. Scissors and sponge vitrectomy is inadequate for dealing even with small strands of vitreous $(57 \%$ had vitreous remaining within the AC post-operatively) and should be confined to iris and wound toilet at the end of the formal vitrectomy. Our results show that in terms of visual acuity the pres-

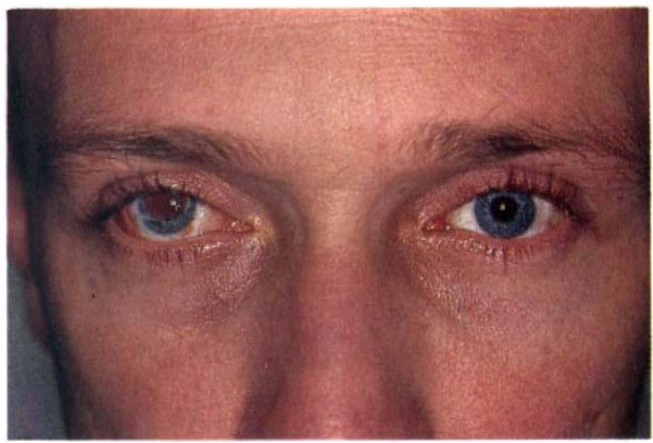

Fig. 10 Same eye as Figure 8 and 9 post squint surgery with adjustable sutures. 


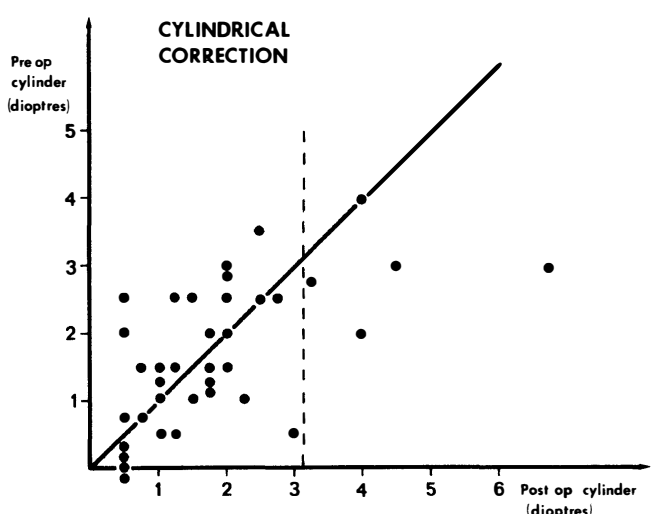

Fig. 11 Difference in cylindrical correction pre- and post-lens implantation.

ence of vitreous in the anterior chamber should not be a contra-indication to secondary lens implantation and others have concurred with this. ${ }^{6,7}$

Although the trauma group had a higher incidence of intense uveitis post-operatively patients in this group responded to intensive topical steroid and then resolved and were not responsible for the high incidence $(21 \%)$ of prolonged uveitis. Persistent and intense uveitis has not been a feature widely reported in other series and where it has been recorded ${ }^{7}$ the incidence has been much lower $(2.6 \%)$. The increase incidence in this series could not be accounted for by the presence of Groups 2 and 3 .

Cases that underwent secondary implantation of a posterior chamber IOL all maintained or improved on their pre-operative visual acuity. However one developed a prolonged uveitis with a requirement for daily topical steroids. This sort of procedure should not be thought of as free of complications. ${ }^{8,9}$

The results of the change in astigmatism underline the importance of correctly placing an incision to avoid an increase in astigmatism. ${ }^{10}$ However other factors such as PAS, broad iridectomies and large peripheral iridectomies may dictate the lie of the lens and hence the axis of incision. Our trauma cases seemed particularly prone to post-operative astigmatic errors and if an incision has to be placed on an axis where there is already a positive cylinder, thought should be given to placing correcting tension sutures at $90^{\circ}$. Although 'aphakic correction +7 dioptres' rule has been used in the past to estimate lens power it has been shown that pre-operative biometry is more accurate. ${ }^{11}$

Our results show the effectiveness of this technique for the maintenance of BSV following extraction of a traumatic cataract. It has been our experience that young people do not persevere with a contact lens in the treatment of unilateral aphakia under these circumstances with consequent secondary divergence. Even if a contact lens is worn control of binocularity may be improved with an intra-ocular lens. ${ }^{12}$

Although there are other methods of visual rehabilitation, such as epikeratophakia, ${ }^{13}$ this technique may not achieve such good visual acuity and the time taken to achieve best VA is longer. In addition, many of these patients will require entry into the anterior chamber to facilitate other anterior segment surgery.

\section{Conclusion}

Even in the disorganised eye this technique gives good results providing that the vitreous is adequately managed.

We should like to thank Dr Susan Bogle for her help in preparing the statistical analysis.

\section{References}

${ }^{1}$ Leatherbarrow B, Trevett A, Tullo AB: Secondary Lens Implantation: Incidence, Indications and Complications. Eye 1988, 2: 370-5.

${ }^{2}$ Aaberg TM: Pars plana vitrectomy for persistent aphakic cystoid macular oedema secondary to vitreous incarceration in the cataract wound. In McPherson A (ed): New and Controversial Aspects of Vitreo-Retinal Surgery. St Louis, CV Mosby 1977; 230-3.

${ }^{3}$ Cozean $\mathrm{CH}$ : A longer view of secondary intra-ocular lens implantation with special emphasis on the role of the vitreous. Am Intra-ocular Implant Soc J 1980, 6: 361-2.

${ }^{4}$ Shammas HJF and Milkie CF: Cystoid macular oedema following secondary lens implantation. Am Intraocul Implant Soc J 1981, 7: 40-2.

${ }^{5}$ Wong SK, Koch DD, Emery JM: Secondary intraocular lens implantation. J Cataract Refract Surg 1987, 13: 17-20.

${ }^{6}$ Mackool RJ: Simultaneous closed vitrectomy and secondary intraocular lens implantation. $A m$ Intraocul Implant Soc J 1981, 7: 233-6.

${ }^{7}$ Lindstrom RJ and Harris WS. Secondary anterior chamber lens implantation. CLAO Journal 1984, 10: $133-6$.

${ }^{8}$ Mazzocco TR, Kratz RP, Davidson B, Colvard DM: Secondary posterior chamber intra-ocular lens implants. Am Intraocul Implant Soc 1981, 7: 341-3. 
${ }^{9}$ Lindstrom RL, Harris WS, Lyle WA: Secondary and exchange posterior chamber lens implantation. Am Intraocul Implant Soc J 1982, 8: 353-6.

${ }^{10}$ Masket S: Temporal incision for astigmatic control in secondary implantation. J Cataract Refract Surg 1986, 12: 179-81.

${ }^{11}$ Summers CG and Lindstrom RL: Secondary IOL power calculations: A compàrison of regression formula and refraction method in accurate predi- cation of emmetropia. Am Intraocul Implant Soc J 1984, 10: 448-51.

${ }^{12}$ Cole MD, Hay A, Eagling EM: Cyclic esotropia in a patient with unilateral traumatic aphakia: a case report. Br J Ophthalmol 1988, 72: 305-8.

${ }^{13}$ Durrie DS, Habrich DL, Dietze TR: Secondary intra-ocular lens implantation vs epikeratophakia for the treatment of aphakia. Am J Ophthalmol 1987, 103: 384-91. 